\title{
Influence of Concentration of Selected Cationic Surfactants on Drug Liberation
}

\author{
Z. VitkovÁ, D. AntonyovÁ, P. HerdovÁ, M. ŽabkA, J. OremusovÁ
}

Faculty of Pharmacy, Commenius University, Bratislava, Slovakia

E-mail: herdova@fpharm.uniba.sk (P. Herdová)

Sci Pharm. 2010; 78: 626

doi:10.3797/scipharm.cespt.8.PDD37

Drug liberation-release from the dosage form is a primary cause of the movement of a drug in the body. Pharmaceutical availability of the drug depends not only on the type of aditives but also on the selected concentration. In this study there are compared influence of the concentration of selected cationic surfactants on the liberation of antiseptic chlorhexidine from the hydrogels prepared on the chitosan basis.

Objectives:

- formulation of hydrogels based on chitosan with chlorhexidine dihydrochloride

- observation and evaluation of the influence of concentrations of two cationic surfactants on liberation of chlorhexidine dihydrochloride

- selection of an apropriate concentration of cationic surfactants

- determination of the $\mathrm{pH}$ value of hydrogels

- design of an optimal composition of hydrogels.

The drug released was determined by using semipermeable membrane on the permeating apparatus. the released amounts were determined by spectrophotomentric method at $254 \mathrm{~nm}$ from $15 \mathrm{~min}$ to 3 hours

Results:

- As to $\mathrm{pH}$ ranged within 5.44-6.14 the prepared hydrogels were suitable for application in stomatology and dermatology.

- The release of chlorhexidine dihydrochloride depended on the presence and type of cationic surfactant and its concentration.

- It was shown that drug liberation increases with increasing lenght of alkyl chain at the $0.1 \%(\mathrm{w} / \mathrm{w})$ concentration

- Ten times reduction of surfactant concentration incured average increse of the released amount of chlorhexidine dihydrochloride by $15 \%(\mathrm{w} / \mathrm{w})$

- The greatest released amount of chlorhexidine dihydrochloride was released from hydrogel of the following composition: $0.1 \%$ chlorhexidine dihydrochloride $+2.5 \%$ chitosan in the environment of lactic acid $+0.01 \%$ tetradecyltrimethylammonium bromide $+10 \%$ glycerol. This hydrogel was chosen as the most appropriate.

This work was supported by the Grant VEGA No. 1/ 0320/ 08 